\title{
Projections of quadrics in finite projective spaces of odd characteristic
}

go back

full screen

close

quit

\author{
Frank De Clerck Nikias De Feyter*
}

\begin{abstract}
The set of points obtained by projecting a quadric from a point off the quadric on a hyperplane has many interesting properties. Hirschfeld and Thas $[12,13]$ provided a characterization of this set, only by means of its intersection pattern with lines. However, their result only holds when the finite field has even order. Here, we extend their result to finite fields of odd order.
\end{abstract}

Keywords: projections of quadrics, sets of points in projective space with prescribed intersection types, internal points of a conic, external points of a conic

MSC 2000: 51E20, 05B25

\section{Introduction}

Let $\mathcal{Q}_{n+1}$ be a nonsingular quadric in a finite projective space $\operatorname{PG}(n+1, q)$, $n \geq 1$. Consider a point $r \notin \mathcal{Q}_{n+1}$, distinct from its nucleus if $n+1$ and $q$ are even, and a hyperplane $\mathrm{PG}(n, q)$ not through $r$. Let $\mathcal{R}_{n}$ be the projection of the quadric $\mathcal{Q}_{n+1}$ from the point $r$ on the hyperplane $\mathrm{PG}(n, q)$. This set has many nice properties (see Section 2).

A set $\mathcal{K}$ of points of $\mathrm{PG}(n, q)$ is said to be a set of class $\left[m_{1}, \ldots, m_{k}\right], 0 \leq m_{1}<$ $\cdots<m_{k} \leq q+1$, if for every line $L,|L \cap \mathcal{K}|=m_{i}$ for some $1 \leq i \leq k$. It is said to be a set of type $\left(m_{1}, \ldots, m_{k}\right)$ if every $m_{i}$ actually occurs for some line $L$. One can similarly define sets of certain class or type with respect to subspaces of dimension $m \geq 1$.

\footnotetext{
*This research was supported by a BOF ("Bijzonder Onderzoeksfonds") grant at Ghent University.
} 
If $q$ is even, $\mathcal{R}_{n}$ is a set of class $\left[1, \frac{1}{2} q+1, q+1\right]$ in $\mathrm{PG}(n, q)$. Sets of class $[1, m, q+1]$, in $\mathrm{PG}(n, q), q$ even and $q>4$, have been classified. See Tallini Scafati [18], Hirschfeld and Thas [12, 13] and Glynn [9] for more details. The case $q=4$ is special, see also Hirschfeld and Hubaut [10] and Hirschfeld, Hubaut and Thas [11]. If $m=\frac{q}{2}+1, q>4$, then a set of type $(1, m, q+1)$ is indeed the projection of a nonsingular quadric.

If $q$ is odd, $\mathcal{R}_{n}$ is a set of class $\left[1, \frac{1}{2}(q+1), \frac{1}{2}(q+3), q+1\right]$ in $\mathrm{PG}(n, q)$. The purpose of this paper is to prove a characterization of $\mathcal{R}_{n}$ for odd $q$, similar to the results in the $q$ even case. In concrete, we classify all sets of class $\left[1, \frac{1}{2}(q+1), \frac{1}{2}(q+3), q+1\right]$ in $\mathrm{PG}(n, q), n \geq 2$ and $q>7$ odd.

Section 2 provides a general view of the set $\mathcal{R}_{n}$. Sets of class $\left[1, \frac{1}{2}(q+1)\right.$, $\left.\frac{1}{2}(q+3), q+1\right]$ in the plane $\mathrm{PG}(2, q), q$ odd, were classified in [5]. We state this result in Section 3. Some of the sets in the plane occur as plane sections of $\mathcal{R}_{n}$, others do not. In Section 4, we show that some combinations of plane sections cannot occur. Section 5 discusses two notions of singularity in sets of class $\left[1, \frac{1}{2}(q+1), \frac{1}{2}(q+3), q+1\right]$. Next, in Section 6 , we cite the classification of projective Shult spaces by Buekenhout and Lefèvre [3] and Lefèvre-Percsy [17]. This result can be seen as a characterization of quadrics, and it is used in this sense in Sections 7 and 8, where we prove our main result.

Main Theorem. Let $\mathcal{K}$ be a set of class $\left[1, \frac{1}{2}(q+1), \frac{1}{2}(q+3), q+1\right]$ in $\mathrm{PG}(n, q)$, $n \geq 2$ and $q>7$ odd. Then one of the following cases occurs.

1. $\mathcal{K}=\mathcal{R}_{n}$.

2. $\mathcal{K}$ is singular. Either $\mathcal{K}$ is the set of all points of $\mathrm{PG}(n, q)$, or $\mathcal{K}$ is a cone with vertex an $m$-space $U$ of $\mathrm{PG}(n, q), 0 \leq m \leq n-2$, and base a nonsingular set of class $\left[1, \frac{1}{2}(q+1), \frac{1}{2}(q+3), q+1\right]$ in an $(n-m-1)$-space $V$ skew to $U$.

3. $\mathcal{K}$ is quasi singular. Then $\mathcal{K}$ consists of $\frac{1}{2}(q+1)$ hyperplanes through a common (n-2)-space $U$ and a nonsingular set, which is of class $\left[1, \frac{1}{2}(q+1)\right.$, $\left.\frac{1}{2}(q+3), q+1\right]$ in a hyperplane $U^{\prime} \supseteq U, U^{\prime}$ distinct from the $\frac{1}{2}(q+1)$ hyperplanes contained in $\mathcal{K}$.

4. $n=2$ and there is a nondegenerate conic $C$ and a point $p \in C$ such that $\mathcal{K}$ is the union of the tangent line $L$ to $C$ at $p$ and the set of internal points of C.

\section{The set $\mathcal{R}_{n}$}

We recall that $\mathcal{Q}_{n+1}$ is a nonsingular quadric in a finite projective space $\mathrm{PG}(n+$ $1, q), n \geq 1$, and that $r \notin \mathcal{Q}_{n+1}$ is a point distinct from its nucleus if $n+1$ and 




Let $\mathcal{K}$ be a set of class $\left[1, \frac{1}{2}(q+1), \frac{1}{2}(q+3), q+1\right]$ in $\mathrm{PG}(n, q), n \geq 3$ and $q>3$, and let $\pi$ be a plane of PG $(n, q)$. Then clearly $\pi \cap \mathcal{K}$ is a set of class $\left[1, \frac{1}{2}(q+1), \frac{1}{2}(q+3), q+1\right]$ in $\pi$. We say that $\pi$ is a plane of type I, II, . if $\pi \cap \mathcal{K}$ is of type I, II, ..., respectively.

If a plane of type IV consists of $\frac{1}{2}(q+1)$ lines, then we call it a plane of type IVa; otherwise we call it a plane of type IVb. If $\pi$ is a plane of type $\mathrm{V}$ and the line $L_{\pi}$ contains $\frac{1}{2}(q-1)$ points of $\mathcal{K}$, other than $p_{\pi}$, then we say that $\pi$ is a plane of type Va; otherwise $\pi$ is said to be a plane of type $\mathrm{Vb}$. If $\pi$ is a plane of type IV or $\mathrm{V}$, then the point $p_{\pi}$ is also called the vertex of $\pi$.

For every $i \in\left\{1, \frac{1}{2}(q+1), \frac{1}{2}(q+3), q+1\right\}$, let $\mathcal{L}_{i}$ denote the set of lines $L$ such that $|L \cap \mathcal{K}|=i$. Table 1 gives useful information on the sets which occur in Theorem 3.1.

The following proposition, the proof of which is trivial, tells which types of planes can occur in the set $\mathcal{R}_{n}$.

Proposition 3.2. Let $\mathcal{Q}_{n+1}$ be a nonsingular quadric in a finite projective space $\mathrm{PG}(n+1, q), n \geq 1, q$ odd. Let $\mathcal{R}_{n}$ be the projection of the quadric $\mathcal{Q}_{n+1}$ from a point $r \notin \mathcal{Q}_{n+1}$ on a hyperplane $\mathrm{PG}(n, q)$, not through $r$. Let $\pi$ be a plane of $\mathrm{PG}(n, q)$, let $p$ be a point of $\mathcal{R}_{n}$, not in $\pi$, and let $W=\langle p, \pi\rangle$. One of the following cases occurs.

1. If $W \cap \mathcal{Q}_{n+1}$ is a nonsingular elliptic quadric, then $\pi \cap \mathcal{T}_{n}$ is a nondegenerate conic $C$, and $\pi$ is of type $\mathrm{I}$, with $C_{\pi}=C$.

2. If $W \cap \mathcal{Q}_{n+1}$ is a nonsingular hyperbolic quadric, then $\pi \cap \mathcal{T}_{n}$ is a nondegenerate conic $C$, and $\pi$ is of type III, with $C_{\pi}=C$.

3. If $W \cap \mathcal{Q}_{n+1}$ is a quadratic cone, then $\pi \cap \mathcal{T}_{n}$ is either the union of two distinct lines or a single point $p$. In the first case, $\pi$ is of type IVb, and in the second case $\pi$ is of type IVa with vertex $p$.

4. If $W \cap \mathcal{Q}_{n+1}$ is the union of two planes $\pi_{1}, \pi_{2}$, then $\pi \cap \mathcal{T}_{n}$ is the line $\pi_{1} \cap \pi_{2}$ and $\pi$ is of type VII.

5. If $W \cap \mathcal{Q}_{n+1}$ is a line $L$, then $\pi \cap \mathcal{T}_{n}=L$ and $\pi$ is of type VI.

6. If $W \cap \mathcal{Q}_{n+1}$ is the plane $\pi$, then $\pi \subseteq \mathcal{T}_{n}$ is of type VII. 


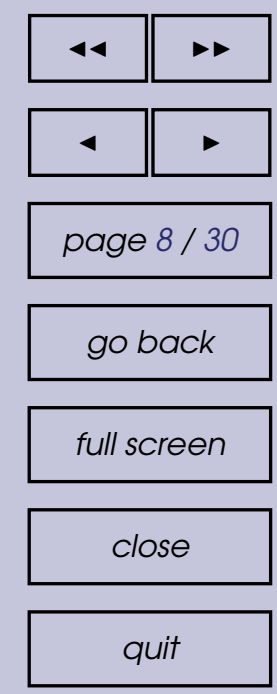

\begin{tabular}{|c|c|c|c|c|c|c|}
\hline$\pi$ & $|\mathcal{K} \cap \pi|$ & $p$ & $\mathcal{L}_{1}$ & $\mathcal{L}_{\frac{1}{2}(q+1)}$ & $\mathcal{L}_{\frac{1}{2}(q+3)}$ & $\mathcal{L}_{q+1}$ \\
\hline$\overline{\mathrm{I}}$ & $\frac{1}{2} q^{2}+\frac{1}{2} q+1$ & $\begin{array}{c}p \in C_{\pi} \\
\text { internal to } C_{\pi} \\
\text { external to } C_{\pi}\end{array}$ & $\begin{array}{l}\mathbf{1} \\
\mathbf{0} \\
2\end{array}$ & $\begin{array}{c}\mathbf{0} \\
\frac{q+\mathbf{1}}{2} \\
\frac{q-1}{2}\end{array}$ & $\begin{array}{c}\boldsymbol{q} \\
\frac{\boldsymbol{q + 1}}{\mathbf{2}} \\
\frac{q-1}{2}\end{array}$ & $\begin{array}{l}\mathbf{0} \\
\mathbf{0} \\
0\end{array}$ \\
\hline II & $\frac{1}{2} q^{2}+\frac{1}{2} q+1$ & $\begin{array}{c}\boldsymbol{p} \boldsymbol{p}=\boldsymbol{p}_{\boldsymbol{\pi}} \\
\boldsymbol{p} \in \boldsymbol{L}_{\boldsymbol{\pi}} \backslash\left\{\boldsymbol{p}_{\boldsymbol{\pi}}\right\} \\
\text { internal to } \boldsymbol{C}_{\boldsymbol{\pi}} \\
p \in C_{\boldsymbol{\pi}} \backslash p_{\pi} \\
\text { external, } p \notin L_{\pi}\end{array}$ & $\begin{array}{l}\mathbf{0} \\
\mathbf{1} \\
\mathbf{0} \\
1 \\
2\end{array}$ & $\begin{array}{c}\boldsymbol{q} \\
\frac{q-1}{2} \\
\frac{q+1}{2} \\
q \\
\frac{q-1}{2} \\
\end{array}$ & $\begin{array}{c}0 \\
\frac{q-1}{2} \\
\frac{q+1}{2} \\
0 \\
\frac{q-1}{2} \\
\end{array}$ & $\begin{array}{l}\mathbf{1} \\
\mathbf{1} \\
\mathbf{0} \\
0 \\
0\end{array}$ \\
\hline III & $\frac{1}{2} q^{2}+\frac{3}{2} q+1$ & $\begin{array}{c}\qquad p \in C_{\pi} \\
\text { external to } C_{\pi} \\
\text { internal to } C_{\pi}\end{array}$ & $\begin{array}{l}\mathbf{0} \\
\mathbf{0} \\
0\end{array}$ & $\begin{array}{c}\mathbf{0} \\
\frac{\boldsymbol{q - 1}}{\mathbf{2}} \\
\frac{q+1}{2}\end{array}$ & $\begin{array}{c}\boldsymbol{q} \\
\frac{\boldsymbol{q - 1}}{2} \\
\frac{q+1}{2}\end{array}$ & $\begin{array}{l}\mathbf{1} \\
\mathbf{2} \\
0\end{array}$ \\
\hline IVa & $\frac{1}{2} q^{2}+\frac{1}{2} q+1$ & $\begin{array}{c}\boldsymbol{p}=\boldsymbol{p}_{\boldsymbol{\pi}} \\
\boldsymbol{p} \neq \boldsymbol{p}_{\boldsymbol{\pi}} \\
p \notin \mathcal{K}\end{array}$ & $\begin{array}{c}\frac{q+1}{2} \\
\mathbf{0} \\
1\end{array}$ & $\begin{array}{l}\mathbf{2} \\
\mathbf{q} \\
q\end{array}$ & $\begin{array}{l}2 \\
\mathbf{0} \\
\mathbf{0} \\
0\end{array}$ & $\begin{array}{c}\frac{q+1}{2} \\
1 \\
0\end{array}$ \\
\hline $\mathrm{IVb}$ & $\frac{1}{2} q^{2}+\frac{3}{2} q+1$ & $\begin{array}{c}\boldsymbol{p}=\boldsymbol{p}_{\boldsymbol{\pi}} \\
\boldsymbol{p} \neq \boldsymbol{p}_{\boldsymbol{\pi}} \\
p \notin \mathcal{K}\end{array}$ & $\begin{array}{c}\frac{q-1}{2} \\
\mathbf{0} \\
1\end{array}$ & $\begin{array}{l}\mathbf{0} \\
\mathbf{0} \\
0\end{array}$ & $\begin{array}{l}\mathbf{0} \\
q \\
q\end{array}$ & $\begin{array}{c}\frac{q+3}{2} \\
1 \\
0\end{array}$ \\
\hline $\mathrm{Va}$ & $\frac{1}{2} q^{2}+q+\frac{1}{2}$ & $\begin{array}{c}\boldsymbol{p}=\boldsymbol{p}_{\boldsymbol{\pi}} \\
\boldsymbol{p} \in \boldsymbol{L}_{\boldsymbol{\pi}} \backslash\left\{\boldsymbol{p}_{\boldsymbol{\pi}}\right\} \\
\boldsymbol{p} \notin \boldsymbol{L}_{\boldsymbol{\pi}} \\
p \in L_{\pi} \\
p \notin L_{\pi}\end{array}$ & $\begin{array}{c}\frac{q-1}{2} \\
\mathbf{0} \\
0 \\
0 \\
1\end{array}$ & $\begin{array}{c}\mathbf{1} \\
\mathbf{1} \\
\frac{q+\mathbf{1}}{\mathbf{2}} \\
q+1 \\
\frac{q+1}{2}\end{array}$ & $\begin{array}{c}\mathbf{0} \\
\boldsymbol{q} \\
\frac{\boldsymbol{q}-1}{2} \\
0 \\
\frac{q-1}{2}\end{array}$ & $\begin{array}{c}\frac{q+1}{2} \\
0 \\
1 \\
0 \\
0\end{array}$ \\
\hline $\mathrm{Vb}$ & $\frac{1}{2} q^{2}+q+\frac{3}{2}$ & $\begin{array}{c}\boldsymbol{p}=\boldsymbol{p}_{\boldsymbol{\pi}} \\
\boldsymbol{p} \in \boldsymbol{L}_{\boldsymbol{\pi}} \backslash\left\{\boldsymbol{p}_{\boldsymbol{\pi}}\right\} \\
\boldsymbol{p} \notin \boldsymbol{L}_{\boldsymbol{\pi}} \\
p \in L_{\pi} \\
p \notin L_{\pi}\end{array}$ & $\begin{array}{c}\frac{q-1}{2} \\
\mathbf{0} \\
0 \\
0 \\
1\end{array}$ & $\begin{array}{c}0 \\
0 \\
\frac{q-1}{2} \\
q \\
\frac{q-1}{2}\end{array}$ & $\begin{array}{c}\mathbf{1} \\
\boldsymbol{q}+\mathbf{1} \\
\frac{\boldsymbol{q + 1}}{2} \\
1 \\
\frac{q+1}{2}\end{array}$ & $\begin{array}{c}\frac{q+1}{2} \\
0 \\
1 \\
0 \\
0\end{array}$ \\
\hline VI & $q+1$ & $\begin{array}{c}p \in \boldsymbol{L}_{\boldsymbol{\pi}} \\
p \notin L_{\pi}\end{array}$ & $\begin{array}{c}\boldsymbol{q} \\
q+1\end{array}$ & $\begin{array}{l}\mathbf{0} \\
0\end{array}$ & $\begin{array}{l}\mathbf{0} \\
0\end{array}$ & $\begin{array}{l}\mathbf{1} \\
0\end{array}$ \\
\hline VII & $q^{2}+q+1$ & $p$ & 0 & 0 & 0 & $q+1$ \\
\hline VIII & 12 & $\begin{array}{c}p \\
p=p_{\pi}^{i} \\
p \neq p_{\pi}^{i}\end{array}$ & $\begin{array}{l}\mathbf{1} \\
4 \\
3\end{array}$ & $\begin{array}{l}4 \\
0 \\
3\end{array}$ & $\begin{array}{l}\mathbf{1} \\
2 \\
0\end{array}$ & $\begin{array}{l}\mathbf{0} \\
0 \\
0\end{array}$ \\
\hline
\end{tabular}

Table 1: For each type of plane $\pi$, the number of points of $\mathcal{K}$ in $\pi$ is given. Also, for all points $p \in \pi$, the number of lines of $\mathcal{L}_{i}$ in $\pi$ through $p$ are given, $i \in\left\{1, \frac{1}{2}(q+1), \frac{1}{2}(q+3), q+1\right\}$. The points $p \in \mathcal{K}$ are printed in bold. 


\section{The projective line}

Let $\mathcal{K}$ be a set of class $\left[1, \frac{1}{2}(q+1), \frac{1}{2}(q+3), q+1\right]$ in $\mathrm{PG}(n, q), n \geq 3, q>3$.

Proposition 4.1. Let $\pi$ be a plane of type I or III. A line $L \subseteq \pi$ is a line of $\mathcal{L}_{\frac{1}{2}(q+1)}$ if and only if $L$ is an external line to the conic $C_{\pi}$. In this case $L \cap \mathcal{K}=\mathcal{R}_{1}^{-}$. On the other hand $L \in \mathcal{L}_{\frac{1}{2}(q+3)}$ if and only if $L$ is a secant line to $C_{\pi}$. In this case $L \cap \mathcal{K}=\mathcal{R}_{1}^{+}$, with $\mathcal{T}_{1}=L \cap C_{\pi}$.

full screen

close

Proof. Proposition 3.2 says that if $\pi$ is of type I, then $\pi \cap \mathcal{K}=\mathcal{R}_{2}^{-}$, and if $\pi$ is of type III, then $\pi \cap \mathcal{K}=\mathcal{R}_{2}^{+}$. Hence Proposition 2.1 applies.

Proposition 4.2. Let $\pi$ be a plane of type II. A line $L \subseteq \pi$ is a line of $\mathcal{L}_{\frac{1}{2}(q+1)}$ if and only if $L$ is a secant line to the conic $C_{\pi}$. In this case $L \cap \mathcal{K}=\mathcal{P}_{1}^{+} \cup\{p\}$, where $\mathcal{T}_{1}=L \cap C_{\pi}$ and $p=L \cap L_{\pi}$. On the other hand $L \in \mathcal{L}_{\frac{1}{2}(q+3)}$ if and only if $L$ is an external line to $C_{\pi}$. In this case $L \cap \mathcal{K}=\mathcal{R}_{1}^{-} \cup\{p\}$, with $p=L \cap L_{\pi}$.

Proof. Note that, when we delete from the set $\pi \cap \mathcal{K}$ the line $L_{\pi}$ and add the conic $C_{\pi}$, then we obtain a set of type I. So we can apply Proposition 4.1.

One can wonder whether the line sections as given in Propositions 4.1 and 4.2 can be projectively equivalent. This question has been discussed (in another context) for instance by J. C. Fisher [8], who proves that $\mathcal{R}_{1}^{-}$and $\mathcal{P}_{1}^{+}$are projectively equivalent if and only if $q \leq 7$. This implies that when $q>7$, planes of type II cannot occur together with planes of type I or III, as we will prove in the next theorem.

Theorem 4.3. Let $\mathcal{K}$ be a set of class $\left[1, \frac{1}{2}(q+1), \frac{1}{2}(q+3), q+1\right]$ in $\mathrm{PG}(3, q), q$ odd. If there is a plane $\pi_{1}$ of type I or III, and a plane $\pi_{2}$ of type II, then $q \leq 7$.

Proof. Suppose that there are such planes $\pi_{1}, \pi_{2}$, and that $q>7$. Let $L=\pi_{1} \cap \pi_{2}$. Let $p \in L$ be a point that is not on the conic $C_{\pi_{1}}$. It follows from Table 1 that there are two lines $L_{1}, L_{2} \in \mathcal{L}_{\frac{1}{2}(q+1)}$ through $p$, distinct from $L$, such that $L_{i} \subseteq \pi_{i}, i=1,2$. Consider the plane $\pi=\left\langle L_{1}, L_{2}\right\rangle$. By Proposition 4.1, $L_{1} \cap \mathcal{K}$ and $L_{2} \cap \mathcal{K}$ are projectively equivalent if $\pi$ is of type I or III. The same holds if $\pi$ is of type IV or V (note that $\pi$ cannot be of type VI, VII or VIII as it contains lines of $\mathcal{L}_{\frac{1}{2}(q+1)}$ and as $q>7$ ). By Proposition 4.1, $L_{1} \cap \mathcal{K}$ is projectively equivalent to $\mathcal{R}_{1}^{-}$, and by Proposition $4.2, L_{2} \cap \mathcal{K}$ is projectively equivalent to $\mathcal{P}_{1}^{+} \cup\left\{p^{\prime}\right\}$ for some point $p^{\prime} \notin \mathcal{P}_{1}^{+}$. As we assume $q>7$, by the result of Fisher, $L_{1} \cap \mathcal{K}$ and $L_{2} \cap \mathcal{K}$ are not projectively equivalent, so $\pi$ is of type II. But then $L_{1} \cap \mathcal{K}$ is projectively equivalent to $\mathcal{P}_{1}^{+} \cup\left\{p^{\prime}\right\}$ for some point $p^{\prime} \notin \mathcal{P}_{1}^{+}$. This contradicts again the result of Fisher. 


\section{5. (Quasi) singularity}

Let $\mathcal{K}$ be a set of class $\left[1, \frac{1}{2}(q+1), \frac{1}{2}(q+3), q+1\right]$ in $\mathrm{PG}(n, q), n \geq 3, q$ odd and $q>7$. For every point $p \in \mathcal{K}$, let $\mathcal{A}_{p}$ be the set of lines $L \in \mathcal{L}_{\frac{1}{2}(q+1)} \cup \mathcal{L}_{\frac{1}{2}(q+3)}$ through $p$. The set $\mathcal{K}$ is called singular if it has a singular point, that is, a point $p$ such that $\mathcal{A}_{p}$ is empty. The set $\mathcal{S}$ of all singular points is always the point set of a subspace of $\mathrm{PG}(n, q)$. Indeed, let $p, r \in \mathcal{S}$. Then every line through $p$ or $r$ is in $\mathcal{L}_{1} \cup \mathcal{L}_{q+1}$. By Table 1, every plane through $L=\langle p, r\rangle$ is of type VI or VII. Hence $L \subseteq \mathcal{S}$. So $\mathcal{S}$ is the point set of an $m$-space $U$ of $\mathrm{PG}(n, q)$. It follows that if $\mathcal{K}$ is singular, then either $m=n$, so $\mathcal{K}$ is the point set of $\operatorname{PG}(n, q)$, or $0 \leq m \leq n-2$ and $\mathcal{K}$ is a cone with vertex $U$ and base a nonsingular set of class $\left[1, \frac{1}{2}(q+1), \frac{1}{2}(q+3), q+1\right]$ in an $(n-m-1)$-space $V$ skew to $U$.

A point $p \in \mathcal{K}$ is called a quasi singular point if $\mathcal{A}_{p} \neq \emptyset$ and $\mathcal{A}_{p}$ is contained in a hyperplane of $\mathrm{PG}(n, q)$. The set $\mathcal{K}$ is called quasi singular if it is not singular and has a quasi singular point.

Lemma 5.1. Let $\mathcal{K}_{1}$ and $\mathcal{K}_{2}$ be sets of class $\left[1, \frac{1}{2}(q+1), \frac{1}{2}(q+3), q+1\right]$ in $\mathrm{PG}(2, q)$, q odd and $q>5$, such that $\mathcal{K}_{1} \neq \mathcal{K}_{2}$ and there is a line $L$ such that $\mathcal{K}_{1} \backslash L=\mathcal{K}_{2} \backslash L$. Then there are lines $L=L_{0}, \ldots, L_{\frac{1}{2}(q+1)}$ through a point $p$ and distinct sets $\mathcal{K}_{1}^{\prime}$ and $\mathcal{K}_{2}^{\prime}$ with $p \in \mathcal{K}_{i}^{\prime} \subseteq L_{0}$ and $\left|\mathcal{K}_{i}^{\prime}\right| \in\left\{1, \frac{1}{2}(q+1), \frac{1}{2}(q+3), q+1\right\}, i=1,2$, such that $\mathcal{K}_{i}=L_{1} \cup \cdots \cup L_{\frac{1}{2}(q+1)} \cup \mathcal{K}_{i}^{\prime}, i=1,2$.

Proof. Let $\mathcal{L}_{i}^{j}$ be the set of lines $M$ such that $\left|M \cap \mathcal{K}_{j}\right|=i, i \in\{1, q+1\}$, $j \in\{1,2\}$. Let $M \neq L$ be a line of $\mathrm{PG}(2, q)$. Since the symmetric difference $\mathcal{K}_{1} \triangle \mathcal{K}_{2} \subseteq L,\left(M \cap \mathcal{K}_{1}\right) \triangle\left(M \cap \mathcal{K}_{2}\right)$ contains at most one point. So if $M \in \mathcal{L}_{i}^{1}$, then also $M \in \mathcal{L}_{i}^{2}$, and moreover $M \cap \mathcal{K}_{1}=M \cap \mathcal{K}_{2}, i=1, q+1$. Using this and Theorem 3.1, it is an easy exercise to prove the lemma.

Proposition 5.2. Let $\mathcal{K}$ be a set of class $\left[1, \frac{1}{2}(q+1), \frac{1}{2}(q+3), q+1\right]$ in $\mathrm{PG}(3, q)$, $q$ odd and $q>5$. If $\mathcal{K}$ has a quasi singular point $p$, then $\mathcal{K}$ consists of the union of $\frac{1}{2}(q+1)$ planes through a line $L \ni p$, together with a set of class $\left[1, \frac{1}{2}(q+1), \frac{1}{2}(q+3), q+1\right]$ in a plane $\pi$ through $L$.

Proof. Let $\pi$ be a plane through $p$ containing the lines of $\mathcal{A}_{p}$. Then $\mathcal{K} \backslash \pi$ is a cone with vertex $p$ and base a set $\mathcal{X}$ in a plane $\pi^{\prime} \not \nexists p$. As $p$ is nonsingular, there exist lines $L_{1}, L_{2}$ in $\pi$, not through $p$, such that the projections $\mathcal{X}_{1}$ and $\mathcal{X}_{2}$ of the sets $L_{1} \cap \mathcal{K}$ and $L_{2} \cap \mathcal{K}$, respectively, from the point $p$ on the line $L^{\prime}=\pi^{\prime} \cap \pi$ are distinct. Let $\pi_{i} \neq \pi$ be a plane through $L_{i}, i=1,2$. As $\mathcal{X} \cup \mathcal{X}_{i}$ is the projection of $\pi_{i} \cap \mathcal{K}$ from $p$ on $\pi^{\prime}, \mathcal{X} \cup \mathcal{X}_{i}$ is a set of class $\left[1, \frac{1}{2}(q+1), \frac{1}{2}(q+3), q+1\right]$ in $\pi^{\prime}, i=1,2$. However $\mathcal{X}_{1} \neq \mathcal{X}_{2}$. It follows from Lemma 5.1 that there are lines $M_{0}\left(=\pi \cap \pi^{\prime}\right), \ldots, M_{\frac{1}{2}(q+1)}$ in $\pi^{\prime}$ through a point $p^{\prime}$ and distinct sets $\mathcal{X}_{1}^{\prime}$ and 











\section{The general result}

In this section, we prove the Main Theorem. Note that for $n=2$ and $n=3$, the Main Theorem holds by Theorems 3.1 and 7.11, respectively. (When $n=2$, a set of type IV, VI or VII is a singular set, and a set of type V is a quasi singular set.) The proof for $n \geq 4$ goes by induction on the dimension of the projective space $\mathrm{PG}(n, q)$.

Consider the quasi singular set $\mathcal{K}$ as in case 3 of the Main Theorem. We say that the $(n-2)$-space $U$ is the vertex of $\mathcal{K}$.

Lemma 8.1. Consider the set $\mathcal{R}_{3}$ in $\mathrm{PG}(3, q)$, q odd. For every line $L \in \mathcal{L}_{\frac{1}{2}(q+1)} \cup$ $\mathcal{L}_{\frac{1}{2}(q+3)}$, there is a plane of type I containing $L$.

Proof. The line $L \in \mathcal{L}_{\frac{1}{2}(q+1)} \cup \mathcal{L}_{\frac{1}{2}(q+3)}$, so it is secant or external to the nonsingular quadric $\mathcal{T}_{3}$. A plane is of type I or III if and only if it intersects the quadric $\mathcal{T}_{3}$ in a nondegenerate conic. Note that every plane is of type I, III or IV.

As $L$ is external or secant to $\mathcal{T}_{3}$, there are at most two planes of type IV through $L$, and these planes contain at least $\frac{1}{2} q^{2}+\frac{1}{2} q+1$ points of $\mathcal{R}_{3}$. Suppose that no plane through $L$ is of type I. Then the other planes through $L$ are of type III. It follows that

$$
\begin{aligned}
\left|\mathcal{R}_{3}\right| & \geq \frac{1}{2}(q+3)+2\left(\frac{1}{2} q^{2}-\frac{1}{2}\right)+(q-1)\left(\frac{1}{2} q^{2}+q-\frac{1}{2}\right) \\
& =\frac{1}{2} q^{3}+\frac{3}{2} q^{2}-q+1 .
\end{aligned}
$$

But this contradicts Proposition 2.2.

Theorem 8.2. Let $n \geq 4$, and suppose that the Main Theorem holds in PG $(m, q)$, $q$ odd and $q>7$, for all $2 \leq m<n$. Let $\mathcal{K}$ be a set of class $\left[1, \frac{1}{2}(q+1), \frac{1}{2}(q+3)\right.$, $q+1]$ in $\mathrm{PG}(n, q), q$ odd and $q>7$. If there is a plane of type II or $\mathrm{V}$, then every subspace of dimension $3 \leq m<n$ intersects $\mathcal{K}$ in a (quasi) singular set.

Proof. If there is an $m$-space $U, 3<m<n$, such that $\mathcal{K} \cap U$ is not (quasi) singular, then by the Main Theorem $\mathcal{K} \cap U=\mathcal{R}_{m}$, so $U$ contains a 3 -space $W$ such that $\mathcal{K} \cap W$ is not (quasi) singular. Hence it suffices to show that every 3 -space intersects $\mathcal{K}$ in a (quasi) singular set.

Suppose there is a plane $\pi$ of type II or $\mathrm{V}$, and a 3 -space $W$ such that $\mathcal{K} \cap W$ is not (quasi) singular. By Theorem $7.11, \mathcal{K} \cap W=\mathcal{R}_{3}$. Note that by Theorem 7.11, and since $\mathcal{R}_{3}$ only has planes of type I, III or IV, every 3 -space $W^{\prime}$ through $\pi$ intersects $\mathcal{K}$ in a (quasi) singular set. So $\pi \nsubseteq W$.

Assume that $\pi$ intersects $W$ in a line $L \in \mathcal{L}_{\frac{1}{2}(q+1)} \cup \mathcal{L}_{\frac{1}{2}(q+3)}$. By Lemma 8.1, there is a plane $\pi^{\prime}$ of type $\mathrm{I}$ in $W$ through $L$. Let $W^{\prime}=\left\langle\pi, \pi^{\prime}\right\rangle$. Then $W^{\prime} \cap \mathcal{K}$ 


We conclude that for every hyperplane $U^{\prime}$ through $U_{0}$, the set $\left(U^{\prime} / p\right) \cap \mathcal{A}_{p}$ is contained in a subspace of codimension zero in $U^{\prime} / p$. Hence

$$
\left|\mathcal{A}_{p}\right| \leq \frac{q^{n-1}-1}{q-1}
$$

Suppose that $\mathcal{K}$ is not (quasi) singular. Then $\mathcal{A}_{p}$ spans $\mathrm{PG}(n, q) / p$. Let $U$ be a hyperplane of $\operatorname{PG}(n, q)$ containing $p$ such that $\mathcal{A}_{p} \cap(U / p)$ spans $U / p$.

If $U \cap \mathcal{K}$ is a cone with vertex an $(n-4)$-space $W$ and base a plane of type I, II or III, then $p \notin W$. Let $\pi \ni p$ be a plane skew to $W$. Table 1 says that there are at least $q-1$ lines of $\mathcal{A}_{p}$ in $\pi$. So $\left|\mathcal{A}_{p} \cap(U / p)\right| \geq q^{n-2}-q^{n-3}$.

If $U \cap \mathcal{K}$ is a cone with vertex an $(n-3)$-space $V$ and base a set of $\frac{1}{2}(q+1)$ or $\frac{1}{2}(q+3)$ points on a line, then $p \notin V$ and $\left|\mathcal{A}_{p} \cap(U / p)\right|=q^{n-2}$.

Suppose that $\mathcal{K} \cap U$ consists of $\frac{1}{2}(q+1)$ subspaces of dimension $n-2$ through an $(n-3)$-space $V$ and a set of class $\left[1, \frac{1}{2}(q+1), \frac{1}{2}(q+3), q+1\right]$ in an $(n-2)$ space $U^{\prime}$ through $V$. Then $p \notin V$ and $\left|\mathcal{A}_{p} \cap(U / p)\right| \geq q^{n-2}$.

We conclude that $\left|\mathcal{A}_{p} \cap(U / p)\right| \geq q^{n-2}-q^{n-3}$ for every hyperplane such that $\mathcal{A}_{p} \cap(U / p)$ spans $U / p$. Using (1) and the fact that $\mathcal{A}_{p}$ spans $\operatorname{PG}(n, q) / p$, a contradiction follows easily.

Lemma 8.4. Consider the set $\mathcal{R}_{n}$ in $\mathrm{PG}(n, q), n \geq 3$, q odd. For every point $p$ of $\mathrm{PG}(n, q)$ and every hyperplane $U \ni p$, there is a plane $\pi$ of type I or III, such that $p \in \pi \nsubseteq U$.

Proof. A plane is of type I or III if and only if it intersects the quadric $\mathcal{T}_{n}$ in a nondegenerate conic. The lemma follows from the fact that $\mathcal{T}_{n}$ is a nonsingular quadric.

Lemma 8.5. Let $n \geq 2$, and suppose that the Main Theorem holds in $\mathrm{PG}(m, q), q$ odd and $q>7$, for all $2 \leq m \leq n$.

Let $\mathcal{K}_{1}$ and $\mathcal{K}_{2}$ be sets of class $\left[1, \frac{1}{2}(q+1), \frac{1}{2}(q+3), q+1\right]$ in $\operatorname{PG}(n, q)$, q odd and $q>7$, such that $\mathcal{K}_{1} \neq \mathcal{K}_{2}$ and there is a hyperplane $U$ such that $\mathcal{K}_{1} \backslash U=$ $\mathcal{K}_{2} \backslash U$. Then there is a set of $\frac{1}{2}(q+1)$ hyperplanes $\mathcal{U}=\left\{U_{1}, \ldots U_{\frac{1}{2}(q+1)}\right\}$ through a common $(n-2)$-space $U^{\prime}$, and sets $\mathcal{K}_{1}^{\prime}$ and $\mathcal{K}_{2}^{\prime}$ of class $\left[1, \frac{1}{2}(q+1), \frac{1}{2}(q+3), q+1\right]$ in a hyperplane $U_{0} \notin \mathcal{U}$ through $U^{\prime}$, such that $\mathcal{K}_{j}=U_{1} \cup \cdots \cup U_{\frac{1}{2}(q+1)} \cup \mathcal{K}_{j}^{\prime}$, $j=1,2$.

Proof. If $n=2$, the lemma holds by Lemma 5.1. Suppose that the lemma holds for all $2 \leq n^{\prime}<n$.

Let $\mathcal{L}_{i}^{j}$ be the set of lines $L$ such that $\left|L \cap \mathcal{K}_{j}\right|=i, i \in\{1, q+1\}, j \in\{1,2\}$. Clearly a line $L \nsubseteq U$ is in $\mathcal{L}_{i}^{1}$ if and only if it is in $\mathcal{L}_{i}^{2}, i \in\{1, q+1\}$. 






